\title{
Fabrication of 3D Nanowire Frames by Conventional Micromachining Technology
}

\author{
E. Sarajlic, E. Berenschot, G. Krijnen, M. Elwenspoek \\ MESA+ Research Institute, University of Twente, The Netherlands \\ e.sarajlic@el.utwente.nl
}

\begin{abstract}
We report on a simple parallel processing method capable of producing addressable three-dimensional (3D) nanometersized structures, such as wires, wire frames and dots. The method, which is fully compatible with standard micromachining, employs isotropic removal of conformally deposited material onto a prepared template, to form nanostructures in the concave corners of the template. The process results in well-defined nanometer scale structures with exact position and spatial artangement fully determined by the template. An etching mask with nanometer size features and a nanowire pyramid on a freestanding cantilever, have been successfully fabricated, demonstrating the feasibility and potential of this technology.
\end{abstract}

Keywords: 3D nanofabrication

\section{INTRODUCTION}

Isotropic etching of thin films conformally deposited on topographic steps clears the deposited material from planar surfaces, leaving a portion of the material along the edges of steps. These etching artifacts, known in IC fabrication as stringers or veils, were previously considered highly undesirable.

In this paper, we exploit the abovementioned artifact to controllably form nanometer scale structures in three dimensions (3D) with well-defined position and spatial arrangement. In the following sections, we introduce the basic steps of the 3D nanofabrication method and prove its feasibility by fabricating an etching mask with nanometer size features and a 3D structure composed of nanowires.

\section{D NANOFABRICATION}

Fig. 1 illustrates the basic processing steps of the proposed 3D nanofabrication method.

The fabrication process starts by creating a template with concave comers $(I)$. A material layer is conformally deposited on the prepared template $(I I)$ and a selective isotropic etch of the deposited layer is performed. The access of the etchant to the material located in the concave corner is restricted by the comer geometry. Well-timed isotropic etching clears the deposited fil $\mathrm{lm}$ from all template surfaces, except the material in the concave corner, resulting in well-defined structures with position and spatial arrangement fully determined by the template (III). To achieve good control over the resulting structures, the etching must be highly isotropic and selective to the underlying material.

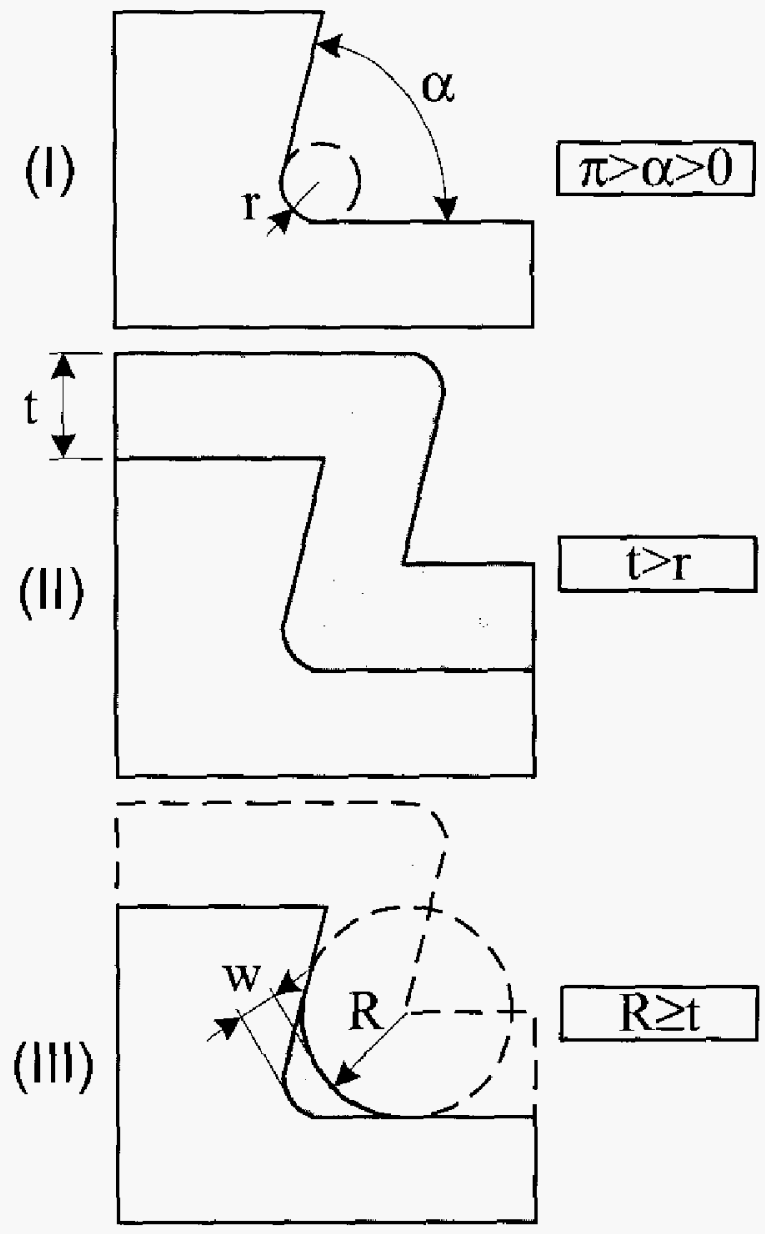

Figure 1: 3-D nanofabrication method: (I) template preparation, (II) conformal deposition and (III) timecontrolled selective isotropic removal.

The characteristic width $w$ of the fabricated structures depends on the angle of the concave comer $\alpha$, its radius of curvature $r$, the thickness $t$ of the deposited layer and the isotropic etching distance $R$,

$$
w=\frac{t-r}{\sin \left(\frac{\alpha}{2}\right)}+r-R
$$


To produce well-defined nanoscale structures, the deposited layer must be uniform, with a good step coverage. Small thickness and good uniformity can be achieved with LPCVD techniques. A thinner deposited layer is favourable, because it allows the fabrication of smaller structures. Nevertheless, the thickness needs to be larger than the radius of curvature $(P r)$. Otherwise, the material from all surfaces, including the concave corners, would be removed simultaneously, resulting in failure of the fabrication process.

The radius of curvature $r$ limits the minimum size of structures that can be formed in the concave comer. Therefore, a template with non-rounded concave comers $(r=0)$ is preferred. Anisotropic etching of silicon in $\mathrm{KOH}$, leads in a straightforward way to concave corners with $r$ smaller than $1 \mathrm{~nm}$. An interesting alternative is to etch silicon by Reactive Ion Etching (RIE) followed by thermal oxidation, which sharpens the resulting concave features down to a radius $r$ a few tens of nm [1], with potential for reaching $<<1 \mathrm{~nm}$. The former method restricts the possible structures to the crystallographic properties of silicon, while the latter gives much more design freedom. Other etching and patterning techniques and template materials can be employed as well.

The etching distance $R$ is determined by the duration and rate of isotropic removal. $R$ needs to be marginally larger than the thickness $t(R>t)$ to remove all the deposited material from planar surfaces. A low etching rate is preferred, because it allows a better control of the structure size.

Comer angle $\alpha$ affects the width of fabricated structures, since material remains longer in corners with smaller angles, resulting in wider structures.

\section{EXPERIMENTS}

In this section, we prove the feasibility and large potential of the $3 \mathrm{D}$ nanofabrication method by creating a silicon oxide etching mask with nanometer size features using conventional photolithography and by fabrication of a pyramid composed of silicon nitride nanowires on a freestanding cantilever beam.

\section{NanoMask}

The basic processing steps for fabrication of a silicon oxide mask with nanometer size features are shown in Fig. 2 .

The fabrication process starts on a single crystal silicon substrate with $\langle 100\rangle$ crystallographic orientation. In the selected substrate we patterned a mold from a rectangular mask using anisotropic etching in $25 \mathrm{wt} \% \mathrm{KOH}: \mathrm{H} 2 \mathrm{O}$ at 75 ${ }^{\circ} \mathrm{C}$ (Step 1 ). The mold is bounded by (111) crystallographic planes that exhibit a very low etch rate in $\mathrm{KOH}$. At the intersection of these planes concave corners with very small radii of curvature are formed, typically less than $1 \mathrm{~nm}$. On the patterned substrate, a low stress silicon nitride layer is conformally deposited by LPCVD, resulting in an uniform $80 \mathrm{~nm}$ thick layer along the substrate surface (Step 2).

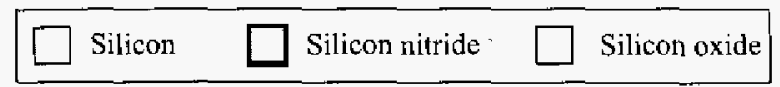

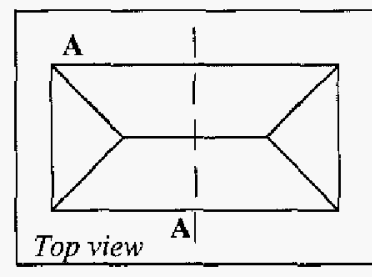

(Step 1)

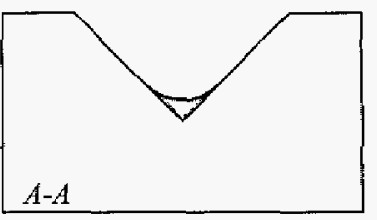

(Step 3 )

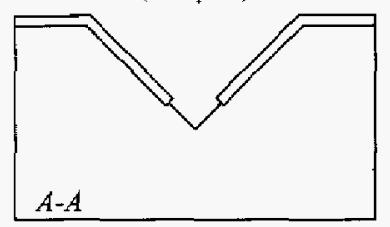

(Step 5)

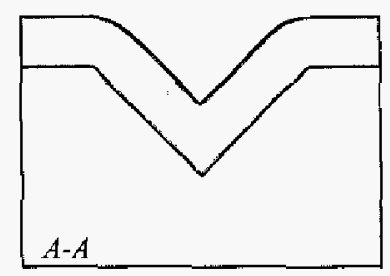

(Step 2)

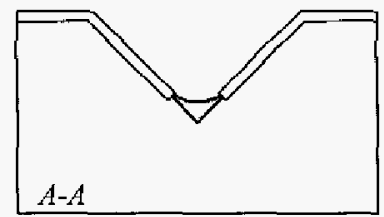

( Step 4)

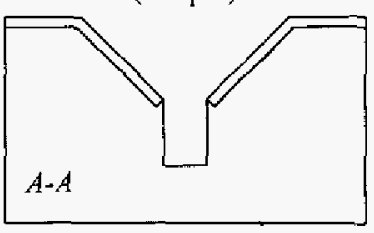

(Step 6)
Figure 2: Processing steps for a silicon oxide etching mask with nanometer-sized features using conventional micormachining and standard lithography.

Next, silicon nitride is etched isotropically in $50 \% \mathrm{HF}$ at room temperature (Step 3). The etching proceeded until the silicon nitride is removed from the top surface of the substrate, indicated by the hydrophilic-hydrophobic transition. Silicon nitride in the concave comers of the mold was preserved in this etching step, as discussed in the previous section. A relatively low etch rate of silicon nitride in HF $50 \%(3.5 \mathrm{~nm} / \mathrm{min})$ makes the end point detection of the etch process less critical, allowing good control of the final nanostructures size. Additionally, the underlying silicon is not affected in the etching process, due to its extremely slow etch rate in HF-based etchants [2]. In the next fabrication step, a $100 \mathrm{~nm}$ silicon oxide layer is thermally grown on the silicon (Step 4). Silicon in the concave comers of the mold is protected from thermal oxidation by the silicon nitride nanostructures. Subsequently, remaining silicon nitride is selectively removed from the concave corners (Step 5). We used an $85 \%$ phosphoric acid $\left(\mathrm{H}_{3} \mathrm{PO}_{4}\right)$ solution at $180^{\circ} \mathrm{C}$, which has a large etch selectivity for silicon nitride compared to silicon oxide and silicon [2]. Removal of the remaining silicon nitride results in a silicon oxide mask with nanometer size openings, located on the concave corners of the silicon mold. Finally, the underlying silicon is etched through the openings in the silicon oxide mask using plasma etching (Step 6). Fig. 3 shows the cross section of a silicon mold after the plasma etching. 


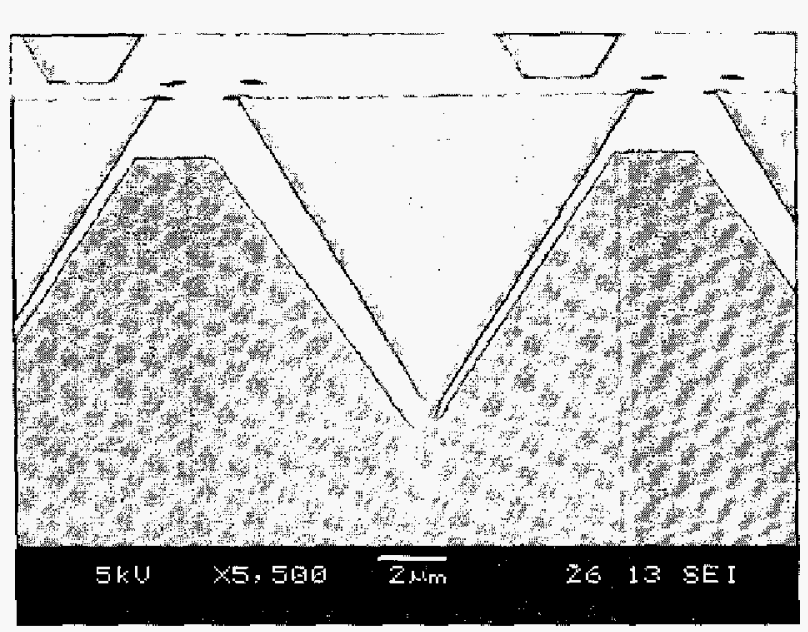

Figure 3: SEM micrograph of a plasma etched silicon template. Concave comers of the template were protected by silicon nitride nanostructures during the oxidation step. After the selective removal of the silicon nitride, openings in the silicon oxide are left, which served as an etching mask for the silicon.

As seen in Fig. 3, the openings in the silicon oxide mask are created at each concave comer regardless of its spatial position or orientation.

\section{Nanopyramid}

In the previous experiment we used the $3 \mathrm{D}$ nanofabrication method to create an etching mask with nanometer features. Here, we present a fabrication process for 3D structures composed of silicon nitride nanowires, which are attached to a freestanding cantilever.

The fabrication process, shown in Fig. 4, starts with deposition of a $500 \mathrm{~nm}$ thick silicon nitride layer on a $<100>$ silicon substrate by LPCVD. In the silicon nitride layer, we pattern a circular opening using directional plasma etching. Subsequently, we form a pyramidal cavity by anisotropic etching of the single crystal silicon in $25 \% \mathrm{KOH}$ solution at $75{ }^{\circ} \mathrm{C}$ (Step 1). The etching proceeds until the (111) crystallographic planes are reached, resulting in the undercutting of the mask. The undercutting forms a concave corner between the sidewalls of the cavity and the silicon nitride layer. Subsequently, we deposit a second low stress silicon nitride layer with a thickness of $80 \mathrm{~nm}$ using LPCVD technique (Step 2). Using 50\% HF, we then isotropically remove this layer from all surfaces with exception of the material in the comers of the pyramidal cavities and between the sidewalls of the cavity and the top silicon nitride layer (Step 3). An unpatterned silicon wafer with a silicon nitride layer of the same thickness is employed to determine the etching time. In the next fabrication step we pattern a cantilever in the first silicon nitride layer by directional plasma etching using a photoresist mask. The silicon nitride nanostructures, formed in the previous step, are protected by the photoresist during the plasma etching. Finally, the cantilever and attached silicon nitride nanowire pyramid are released by front side etching of the silicon in $25 \% \mathrm{KOH}$ solution at $75^{\circ} \mathrm{C}$ solution (Step 4).

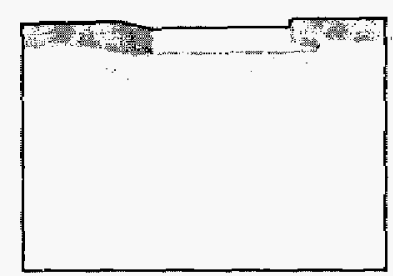

(Step 1)

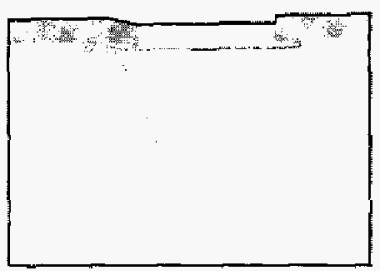

(Step 3)

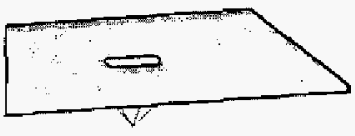

3D Model
(Step 2)

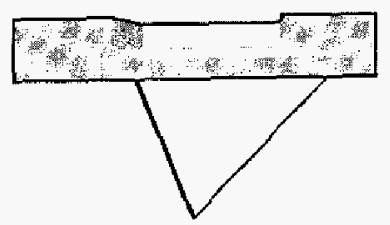

(Step 4)

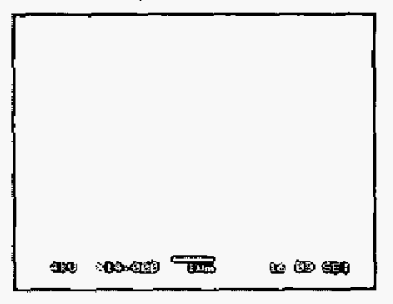

SEM micrograph

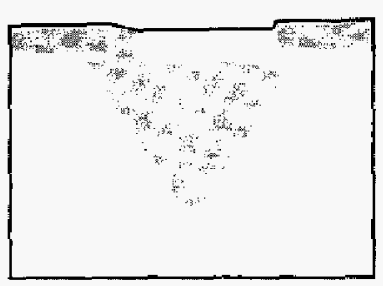

Figure 4: Processing steps for a $3 D$ pyramid composed of silicon nitride nanowires, attached on a freestanding cantilever beam.

A SEM micrograph of the pyramid is shown in Fig. 5. As expected from the theory, the thickness of the nanostructures is determined by the geometry of the concave comers, with smaller angles resulting in thicker structures. Therefore, nanowires formed at the intersection of the sidewalls of the pyramidal cavity and the silicon nitride layer $\left(57.4^{\circ}\right)$ are thicker than those formed at the intersection of two sidewalls $\left(90^{\circ}\right)$. At the intersection of more than two planes, the structure is even thicker, as shown in Fig. 5 at the apex or base of the pyramid.

\section{DISCUSSION AND CONCLUSIONS}

We have presented a simple parallel processing method based on conventional photolithography that produces welldefined nanometer scale structures in three dimensions. The method is based on thin film deposition and selective isotropic removal of material from a prepared template.

The basic requirements of a successful process are: (a) a template with concave corners with radii of curvature smaller than the film thickness, (b) conformal coverage of the template with thin film, (c) isotropic removal of the thin film and (d) a selective etch process. In principle any combination of materials complying with the four basic requirements may be used in the proposed process. We expect that nanostructures can be fabricated.from a variety of materials, including metals. 


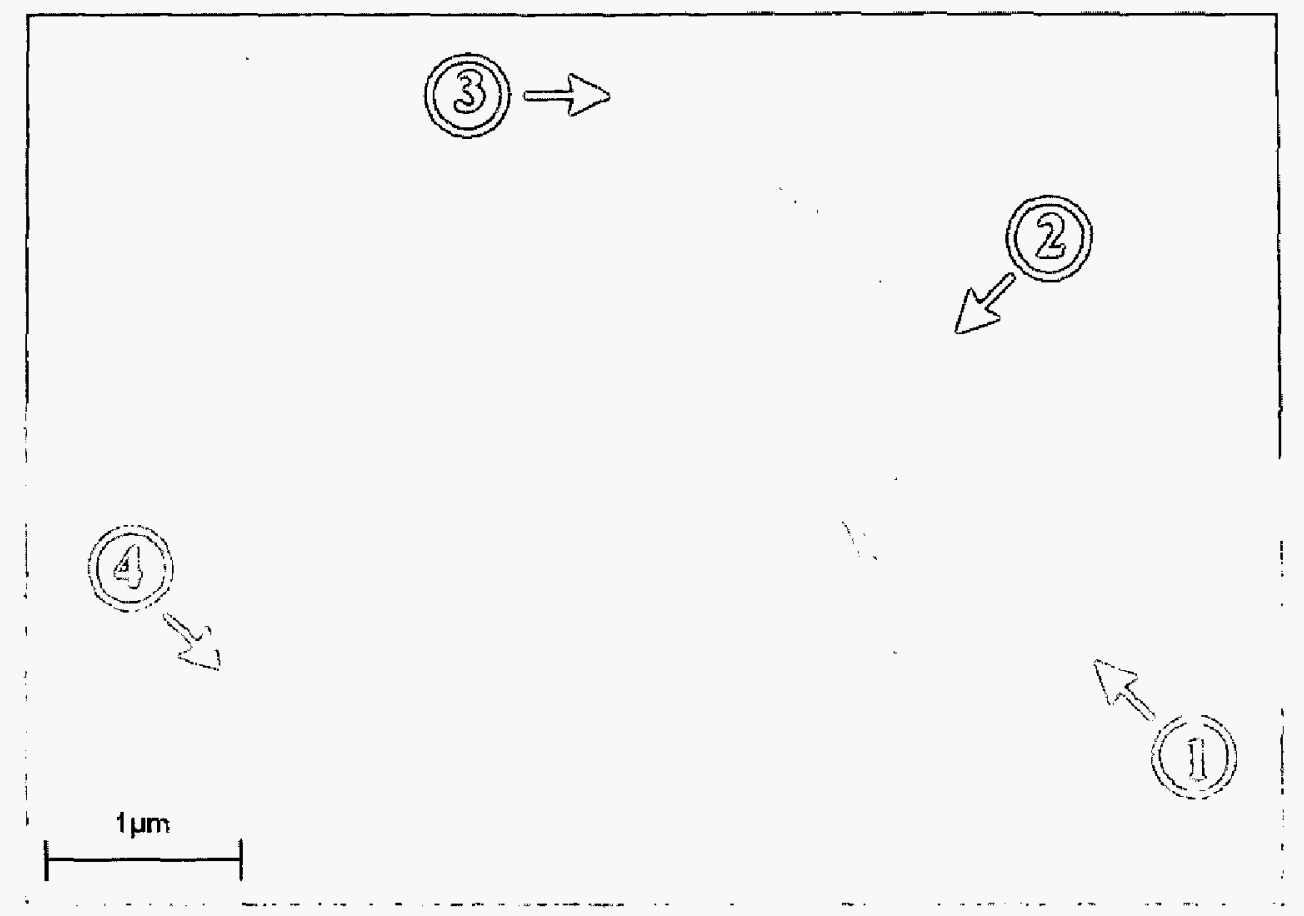

Figure 5: SEM micrograph of a pyramid composed of silicon nitride nanowires, integrated on a cantilever beam. Different geometry of concave angles determines the resulting thickness of the wires: 1 . Two planes intersect at $57.4^{\circ}$ angle; 2. Two planes intersect at $90^{\circ}$ angle; 3. Four planes intersect; 4. Three planes intersect.

Dimensions of the nanostructures in the range of a few nanometers are feasible (see Fig. 6) by controlling the geometry of the concave corners, the thickness of the deposited thin film and the duration and the rate of isotropic removal.

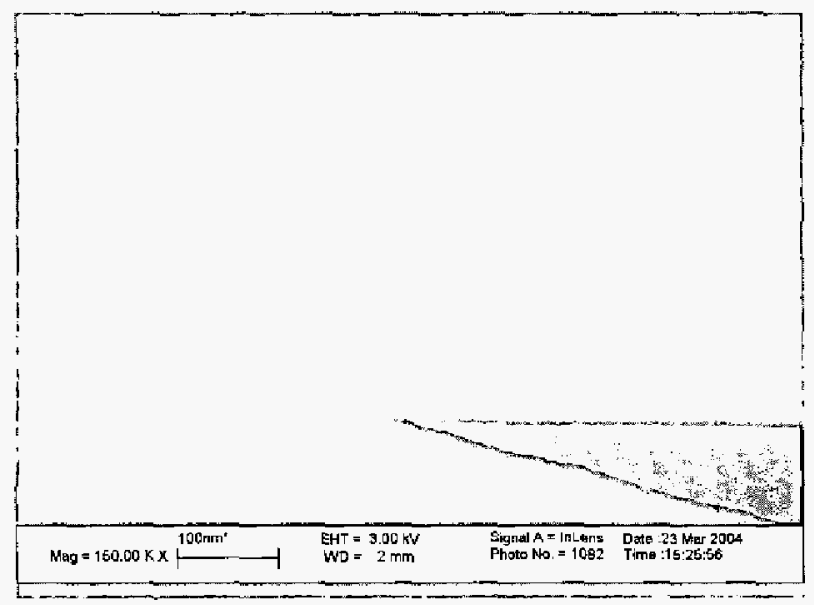

Figure 6: SEM micrograph of a protruding $17 \mathrm{~nm}$ thick silicon nitride structure.

The 3D nanofabrication method can be employed in a varicty of ways. The nanostructures, defined in this process, can be used as a mask to pattern the underlying substrate or layer, producing nanometer features with standard micrometer lithography (see Fig. 3) Additionally, complex $3 \mathrm{D}$ structures composed of nanowires can be fabricated and integrated with other functional components such as bond pads, cantilever beams, membranes and bridges, complex spring constructions, microactuators and sensors, ete. A pyramid composed of silicon nitride nanowires, suspended on a freestanding cantilever shown in Fig. 5, illustrated this potential. The fabrication of dots is an extension of this process. Inverted tips, an example is the apex of the pyramid in Fig. 5, are formed when three or more planes of the template meet. The material in the inverted pyramid tip is thicker than the edges, and will resist etching longest, resulting in a nanodot. Furthermore, nanowires can be covered by a third material, and then selectively removed. In this way, complex nanotube systems can be fabricated and easily connected to micro-sized functional structures (reservoirs, flow sensors, pressure sensors, electrodes for EOF flow, etc.).

Advantages of this new top-down approach to the fabrication of 3-D structures include the simplicity of the process, compatibility with conventional micromachining, straightforward use of photolithography, the possibility to fabricate three-dimensional structures in a wide range of materials and addressability of these structures by other functional components.

\section{REFERENCES}

[1] Marcus R B, Ravi T S and Gmitter T 1990 Formation of silicon tips with $<1 \mathrm{~nm}$ radius Appl. Phys, Left. 56(3) $236-$ 238

[2] Williams K R 1996 Etch rates for micromachining processing J. Microelectromech. Syst. 5(4) 256-269

\section{TRANSDUCERS'05}

The 13th International Conference on Solid-State Sensors, Actuators and Microsystems, Scoul, Korea, June 5-9, 2005 